\title{
Comments on Lightlike Translations and Applications in Relativistic Quantum Field Theory
}

\author{
W. Driessler \\ Department of Theoretical Physics, University of Bielefeld, D-4800 Bielefeld, \\ Federal Republic of Germany
}

Received March 8, 1975; in revised form April 28, 1975

\begin{abstract}
In the algebraic framework of quantum field theory we consider one parameter subgroups of lightlike translations. After establishing a few preliminary properties we prove a certain cluster property and then exhibit the close connection between such subgroups and a class of type III factors. A few applications of this connection are also discussed.
\end{abstract}

\section{Introduction}

When looking for applications of spectrum condition together with locality in relativistic QFT one finds that up to this time the possibilities of using lightlike translation subgroups of the Poincaré group in connection with geometric properties of Minkowski space have not yet been fully realized.

In the present work we shall argue that, roughly speaking, "thinking in lightlike terms" may be useful not only in elementary particle physics but also in general relativistic QFT leading to

a) new proofs and strengthened versions of existing results,

b) entirely new results which would be specific for relativistic theories and which could probably not be obtained otherwise.

In Section 1 we formulate our assumptions and state a few preliminary results on lightlike translations in field theory, some of which will not be used in the sequel of this work but might be useful in further studies.

In Section 2 we prove the main theorem, i.e. by use of the properties of lightlike translations we show that the von Neumann algebras belonging to certain unbounded regions are always type III factors.

The applications (discussed in Section 3) are not very strong by themselves and should be judged more from the methodological point of view; we hope, however, that they will be sufficient to convince the reader that the role of lightlike translations in relativistic field theory deserves more thorough investigation.

\section{Lightlike Translations in Relativistic Field Theory}

We shall work in the algebraic framework of QFT and assume:

a) $\mathfrak{A}$ is a $C^{*}$-algebra of quasilocal operators in a separable Hilbert space $\mathscr{H}$ and is generated in the usual way by local von Neumann algebras $\mathfrak{R}(\mathcal{O})$ which belong to bounded open regions (b.o.r.) in Minkowski space $\mathbb{R}^{n}(n \geqq 2)$ and are subject to locality and isotony. 
$\beta)$ We have a strongly continuous homomorphism $\tau:(\boldsymbol{a}, \Lambda) \rightarrow U(\boldsymbol{a}, \Lambda)$ of $\mathscr{P}_{+}^{\text {? }}$ into the set of unitary operators in $\mathscr{H}$ such that

$$
U(\boldsymbol{a}, \Lambda) \mathfrak{R}(\mathcal{O}) U(\boldsymbol{a}, \Lambda)^{-1}=\mathfrak{R}\left(\mathcal{O}_{\boldsymbol{a}, \Lambda}\right)
$$

$\left(\mathcal{O}_{a, \Lambda}\right.$ denotes the image of $\mathcal{O}$ when $\mathscr{P}_{+}^{r}$ acts as transformation group on $\left.\mathbb{R}^{n}\right)$; so each $U(\boldsymbol{a}, \Lambda)$ induces a $*$-automorphism on $\mathfrak{A}$.

$\gamma)$ There is exactly one vector $\Omega$ in $\mathscr{H}$ which is invariant with respect to time translations $U(t), t \in \mathbb{R} ; \Omega$ is a cyclic vector for $\mathfrak{Q}$.

$\delta)$ Spectrum condition with a mass gap holds for the translation operators $\{U(\boldsymbol{a})\}_{\boldsymbol{a} \in \mathbb{R}^{n}}$ in $\mathscr{H}$.

\&) We have weak additivity for every algebra $\mathfrak{R}(\mathcal{O})$.

By $\delta) \Omega$ is invariant with respect to all translations, therefore also with respect to all $U(\boldsymbol{a}, \Lambda)$. The assumptions imply irreducibility of $\mathfrak{U}$ in $\mathscr{H}$ and the Reeh Schlieder theorem for all algebras $\mathfrak{R}(\mathcal{O})$ ([1], Theorems IV.1.5 and IV.1.6). Since all assumptions are standard they need not be discussed in detail.

Once and for all we fix a lightlike direction - determined by the vector $(1,-1.0 \ldots 0)-($ no loss of generality by rotation invariance); then we consider the one parameter unitary group of translations in this direction $\{U(a)\}, a \in \mathbb{R}$. By Stone's theorem this group has a selfadjoint generator $P_{\|}$; the properties of this operator which are relevant to our purpose are gathered in:

Theorem 1.1. We have: a) $P_{\|} \geqq 0$ in $\mathscr{H}$; b) $\Omega$ is the only vector satisfying: $P_{||} \Omega=0$; c) the spectrum of $P_{\|}$in $\Omega^{\perp}-$ the orthogonal complement of $\Omega$ in $\mathscr{H}-i s$ absolutely continuous, i.e. we have:

$$
w-\lim _{|a| \rightarrow \infty} U(a)=P_{\Omega}
$$

where $P_{\Phi}$ denotes the projection onto the vector $\Phi \in \mathscr{H}$.

Assertion a) is a trivial consequence of spectrum condition; b) can be extracted from the methods used in the proof of the first lemma in the appendix of [2]; for c) see e.g. [12]. A straightforward proof of all assertions will also be given in $[3]$.

A first aspect of the particular properties of lightlike translations in Minkowski space - demonstrating that these translations inherit properties not only from the timelike side (positivity of $P_{\|}$) but also from the spacelike side - is revealed in:

Proposition 1.2. The group $\{U(a)\}_{a \in \mathbb{R}}$ acts in a weakly asymptotically abelian manner on $\mathfrak{U}$, i.e. we have for all $A \in \mathfrak{U}$ :

$$
w-\lim _{|a| \rightarrow \infty} U(a) A U(-a)=(\Omega, A \Omega)=: \omega(A) .
$$

Proof. Let $A$ be a local operator and consider the uniformly bounded set of operators $\mathfrak{N}:=\{U(a) A U(-a)-\omega(A)\}_{a \geq 0}$. There exists a b.o.r. $\tilde{\mathcal{O}} \subset \mathbb{R}^{n}$ such that all elements of $\mathfrak{N}$ commute with all $B \in \mathfrak{R}(\tilde{\mathcal{O}})$. Therefore:

$$
\begin{aligned}
& w-\lim _{a \rightarrow+\infty}\{[U(a) A U(-a)-\omega(A)] B \Omega\} \\
= & w-\lim _{a \rightarrow+\infty}\{B[U(a) A U(-a)-\omega(A)] \Omega\}=0
\end{aligned}
$$


the last step resulting from the previous theorem. From density of $\mathfrak{R}(\tilde{\mathcal{O}}) \Omega$ in $\mathscr{H}$ we get:

$$
w-\lim _{a \rightarrow+\infty} U(a) A U(-a)=\omega(A) .
$$

For $a \rightarrow-\infty$ the argument goes similar. Since every operator in $\mathfrak{A}$ can be approximated in norm by local ones the statement follows.

Remark 1.3. In the proof we have used assumption $\varepsilon$ ) via the Reeh Schlieder theorem; this assumption is really not needed; see Lemma 2.4 below.

Remark 1.4. It would be interesting to characterize the lightlike translations as automorphisms of $\mathfrak{A}$ in a representation independent way - as possible with spacelike translations which act in norm asymptotically abelian manner on $\mathfrak{U}$ ([1], Chapter IV). But at present we can do no better than Proposition 1.2 because we need the boosts in proving absolute continuity of $P_{\|}$.

In any case we learn that the subgroup $\{U(a)\}_{a \in \mathbb{R}}$ inherits a certain clustering property from the spacelike translations which though notably weaker than norm asymptotic abelianness - might, together with positivity of $P_{\|}$, be useful in investigating properties of representations of $\mathfrak{A}$ subject to $\alpha)-\varepsilon$ ).

\section{The Main Theorem}

An old problem of algebraic QFT consists in determining which kind of implications the axioms of the theory have on the algebraic structure of the local von Neumann algebras.

Although it is known that the local algebras of e.g. free relativistic scalar fields are in general type III factors $([4,5])$ - for the notion of type of von Neumann algebras see the brief account in [1], Section II, 1f - the abovementioned problem remains largely open up to now, apart from the question of finiteness $([6,7])$.

In this respect the theorem proved below has an interest in its own in establishing a connection of this type for a class of algebras characterized by a simple geometric condition.

In order to introduce this condition we first state:

Definition 2.1. Let $\mathcal{O}$ be open in $\mathbb{R}^{n}$ with nonvoid causal complement $\mathcal{O}^{\prime}$ and let it be shaped in such a way that - possibly after a suitable rotation - exactly one of the following two conditions is satisfied with respect to our lightlike group $\{U(a)\}_{a \in \mathbb{R}}$ :

$$
\begin{array}{lll}
\left.L^{+}\right) \mathcal{O}_{a} \supset \mathcal{O}_{b} & \text { for } & a>b \\
\left.L^{-}\right) \mathcal{O}_{a} \subset \mathcal{O}_{b} & \text { for } & a>b .
\end{array}
$$

Such a $\mathcal{O}$ must clearly be unbounded and as usual we define the von Neumann algebra $\mathfrak{R}(\mathcal{O})$ to be: $\left(\bigcup_{\tilde{\mathcal{O}} \subset \mathcal{O}} \mathfrak{R}(\tilde{\mathcal{O}})\right)^{\prime \prime}$ where $\tilde{\mathcal{O}}$ runs through all b.o.r. contained in $\mathcal{O}$. $L^{+}$) and $L^{-}$) immediately carry over to the algebras $\mathfrak{R}(\mathcal{O})$ by isotony. Regions of this type as well as their corresponding algebras will be called "lightlike monotone"; the set of all such $\mathfrak{R}$ in our Hilbert space $\mathscr{H}$ will be denoted $\mathscr{A}$.

A simple example of such a set is:

$\mathcal{O}_{0}:=\left\{\boldsymbol{x} \in \mathbb{R}^{n} ;\left|x_{0}\right|<\left|x_{1}\right| ; x_{1}<0\right\}$. 
After this definition we state our main:

Theorem 2.2. All lightlike monotone algebras are type III factors, more specifically they are type $I I I_{1}$ factors in the terminology of [10].

Proof. 1) Let $\mathfrak{R} \in \mathscr{A}$ and assume first that $Q$ is a nontrivial projection in the center $\mathfrak{Z}(\mathfrak{R})$ of $\mathfrak{R}$. Then we see that by the very construction of lightlike monotone algebras we must have:

$$
(\Omega, Q U(a) Q \Omega)=(\Omega, Q U(-a) Q \Omega)
$$

for all $a \in \mathbb{R}$ : assume e.g. $L^{-}$) to be satisfied (this is the case for $\mathfrak{R}\left(\mathcal{O}_{0}\right)-$ in case of $L^{+}$) the argument goes the other way round), then for $a<0$ we have $Q_{a} \in 3\left(\Re_{a}\right)$ and thus it commutes with $Q$, because $\mathfrak{R}_{a} \supset \mathfrak{R}$. For all $a>0$, however, we have $Q \in \mathfrak{R}_{a}^{\prime} \supset \mathfrak{R}^{\prime}$ and (2.2) holds, too. Positivity of $P_{\|}$yields a bounded entire function and Liouville's theorem gives:

$$
\|Q \Omega\|^{2}=|(\Omega, Q \Omega)|^{2} .
$$

By construction of $\mathfrak{R}$ and the Reeh Schlieder theorem $\Omega$ is cyclic for $\mathfrak{R}^{\prime}$ so we get from (2.3): $Q=0$ or $Q=1$, a contradiction. Thus $\mathfrak{R}$ is a factor.

2) The type III property of $\mathfrak{R}$ could possibly be proved by using more classical methods than we shall do, but the preceding argument immediately suggests application of the Tomita Takesaki theory [9]. According to this theory the vector $\Omega$ determines a one parameter group of automorphisms of $\mathfrak{R}$, namely the corresponding modular group $\Sigma=\left\{\sigma_{t}\right\}_{t \in \mathbb{R}}$.

The subalgebra $\mathfrak{R}^{\Sigma}$ of $\mathfrak{R}$ which consists of all elements in $\mathfrak{R}$ which are invariant with respect to $\Sigma$ contains precisely those elements $B \in \mathfrak{R}$ which fulfil:

$$
(\Omega, B A \Omega)=(\Omega, A B \Omega)
$$

for all $A \in \mathfrak{R}$ ([8], Theorem 3.6). But this formula immediately shows that the reasoning following (2.2) may be repeated literally with $Q \in \mathcal{3}(\mathfrak{R})$ replaced by $B \in \mathfrak{R}^{\Sigma}$ because with respect to the invariant vector $\Omega$ the statement $B \in \mathfrak{R}^{\Sigma}$ is equivalent to $U(a) B U(-a) \in[U(a) \Re U(-a)]^{\Sigma}$. So we have $\mathfrak{R}^{\Sigma}=\mathbb{C} \cdot \mathbb{1}$.

3) Assume now $\Re$ to be semifinite, i.e. not type III. This is equivalent to saying that all automorphisms $\sigma_{t}$ are inner ones ([9], Corollary 14.2). This in turn means that the Connes invariant $T(\mathfrak{R})([10]$, Chapter 1$)$ is equal to all of $\mathbb{R}$. For $t \in T(\mathfrak{R})$ we get on the other hand by Theorem 1.3.2a) and c) in [10] that $\sigma_{t}$ is implemented by a unitary $u_{t}$ in the center of $\mathfrak{R}^{\Sigma}$, in our case this would mean that all $\sigma_{t}$ are trivial by $\mathfrak{R}^{\Sigma}=\mathbb{C} \cdot \mathbb{1}$. But this would mean that all elements $A \in \mathfrak{R}$ are invariant with respect to $\Sigma$, a contradiction. So $\mathfrak{R}$ must be type III.

4) According to the classification theory of Connes [10], the class of type III factors is divided into subclasses $\mathrm{III}_{\lambda}, \lambda \in[0,1]$. First by [10], Corollary 3.2.5a) we get for the second Connes invariant $S(\mathfrak{R}): S(\mathfrak{R})=$ spectrum $(\Delta)$ with $\Delta$ the modular operator belonging to $\Sigma$. From Theorem 4.2.6b) and c) we then see that $\mathfrak{R}$ cannot be in one of the classes $\operatorname{III}_{\lambda}, \lambda \in(0,1)$; finally Theorem 5.2.1a) shows that $\mathfrak{R}$ cannot be in class $\mathrm{III}_{0}$ either, so $\mathfrak{R}$ is class $\mathrm{III}_{1}$. q.e.d.

This theorem requires various comments:

A) The statement $\mathfrak{R}^{\Sigma}=\mathbb{C} \cdot \mathbb{1}$ in fact implies much more for $\mathfrak{R}$ than just the type III $_{1}$ property. 
We mention only one simple corollary - an easy consequence of the Radon Nikodym theorem for von Neumann algebras ([8], Theorem 5.12):

Corollary 2.3. For any $\mathfrak{R} \in \mathscr{A}$ the only normal weight on $\mathfrak{R}$ invariant with respect to $\Sigma$ is the state $\varrho_{\Omega}$.

This corollary demonstrates that a Poincare invariant vacuum is in some sense a very "extremal" object.

B) Note that in the proof of the theorem we only needed $\Omega$ to be cyclic for $\Re^{\prime}$. Therefore the theorem holds true for any algebra of the form:

$$
\mathfrak{R}_{\mathfrak{M}}:=\left(\bigcup_{a \geqq 0} \mathfrak{M}_{a}\right)^{\prime \prime},
$$

where $\mathfrak{M}$ is any finite or infinite set of operators in some $\mathfrak{R}(\tilde{\mathcal{O}}), \tilde{\mathcal{O}}$ any b.o.r. in $\mathbb{R}^{n}$. This remark emphasizes the geometric content of the theorem.

C) We give two generalizations of the theorem. The first one consists in taking into account superselection. To this end we adopt the situation as described in [11], where each $\mathfrak{R}(\mathcal{O})$ is contained in some field algebra $\mathfrak{F}(\mathcal{O})$; the field algebras being subject to the usual requirements. Then the projection $Q$ onto $\overline{\mathfrak{A} \Omega}$ may be nontrivial; but since $Q \in \mathfrak{R}^{\prime}$ for all $\Re \in \mathscr{A}$ we see that $Q$ has central support $I$ in $\mathfrak{R}^{\prime}$ by the Reeh Schlieder theorem for the $\mathfrak{F}(\mathcal{O})$ - provided we have the usual statistics, i.e. $\mathfrak{R}\left(\mathcal{O}_{1}\right) \subset \mathfrak{F}\left(\mathcal{O}_{2}\right)^{\prime}$ whenever $\mathcal{O}_{1}$ is spacelike to $\mathcal{O}_{2}: \mathcal{O}_{1} \times \mathcal{O}_{2}$. So the reduced algebra $\Re_{Q}([16], \S 1.2 .1)$ is isomorphic to $\mathfrak{R}$ for all $\mathfrak{R} \in \mathscr{A}$, so everything is reduced to the vacuum sector. The second point consists in dropping $\varepsilon$ ) from the assumptions altogether thus emphasizing the predominant role of spectrum condition in our argument. We show that without $\varepsilon) \Omega$ is nevertheless separating for a large number of $\mathfrak{R} \in \mathscr{A}$. This follows from:

Lemma 2.4. With assumptions $\alpha)-\delta) \Omega$ is separating and cyclic for $\mathfrak{R}(\mathcal{O}) \in \mathscr{A}$ if $\mathcal{O}$ and $\mathcal{O}^{\prime}$ both contain balls of arbitrarily large radius.

The proof of this lemma can be given analogous to the one of the second lemma in the appendix of [2].

Remark 2.5. The reader should note a marked difference between the timelike and the lightlike case. Namely if we generate a timelike $\mathfrak{R}$ starting from a finite family $\mathfrak{M}$ of operators in some $\mathfrak{R}(\tilde{\mathcal{O}})$ we will not in general obtain a factor as in the lightlike case.

If, however, a factor is obtained then it is type I by [13], Theorems 1 and 2 .

\section{Applications}

In this section we shall concentrate on one particular aspect, namely we ask what kind of information can be obtained from the known structure of the $\mathfrak{R}$ 's about the local algebras $\mathfrak{R}(\mathcal{O})$. We shall later make use of the fact that Theorem 2.2 provides us with a kind of "Misra condition" ([14], Definition 12), i.e. every $\mathfrak{R}(\mathcal{O})$ may be embedded in some type III factor, the obvious choice being

$$
\mathfrak{R}_{+}:=\left(\bigcup_{a \geqq 0} \mathfrak{R}\left(\mathcal{O}_{a}\right)\right)^{\prime \prime} .
$$


That in our case $\mathfrak{R}_{+}$does not belong to a bounded region containing $\mathcal{O}$ is compensated for by our knowledge that $\mathfrak{R}_{+}$is type III and that it may be chosen to have 10 at its "edge".

First, however, let us point out a few methodological clues by strenghtening a well known result $([6,7])$, namely we prove:

Theorem 3.1. With assumptions $\alpha$ )- $\delta$ ) the local algebras cannot be finite if we merely assume that no $\mathfrak{R}(\mathcal{O})$ is trivial.

Remark 3.2. If $\varepsilon$ ) is assumed, too, the theorem becomes an almost trivial consequence of the main theorem because " $\mathfrak{R}(\mathcal{O})$ finite and $\Omega$ cyclic for $\mathfrak{R}(\mathcal{O})$ " implies that $\mathfrak{R}(\mathcal{O})^{\prime}$ is finite, too ([16], Exercise III.2, No. 2); but for bounded $\mathcal{O} \subset \mathbb{R}^{n} \mathfrak{R}(\mathcal{O})^{\prime}$ contains elements of the set $\mathscr{A}$, a contradiction as every subalgebra of a finite algebra must be finite.

We shall now prove the same thing without using $\varepsilon$ ); thus adding to a clarification of the relation between non-finiteness of the $\mathfrak{R}(\mathcal{O})$ and our general assumptions - in [6] and [7] $\varepsilon$ ) was essential via use of the Reeh Schlieder theorem. We show that finiteness of the $\mathfrak{R}(\mathcal{O})$ contradicts $\mathfrak{R}^{\Sigma}=\{\mathbb{C} \cdot \mathbb{1}\}$ for appropriate $\mathfrak{R} \in \mathscr{A}$; therefore the proof indicates that also such comparatively subtle properties of the $\mathfrak{R}$ 's may be useful in QFT.

Proof of Theorem: 1) Assume there is a b.o.r. $\mathcal{O}$ in $\mathbb{R}^{n}$ such that $\mathfrak{R}(\mathcal{O})$ is finite; then for all b.o.r. $\tilde{\mathcal{O}} \subset \mathcal{O} \mathfrak{R}(\tilde{\mathcal{O}})$ is finite, too. Fix a translate $\tilde{\mathfrak{R}}$ of $\mathfrak{R}\left(\mathcal{O}_{0}\right)$ contained in $\mathfrak{R}(\mathcal{O})^{\prime}$; by Lemma $2.4 \Omega$ is cyclic for this $\tilde{\mathfrak{R}}$. Again let $Q$ denote the projection onto $\overline{\mathfrak{R}(\mathcal{O}) \Omega}$, i.e. $Q \in \mathfrak{R}(\mathcal{O})^{\prime}$ and $Q \Omega=\Omega$; we see that $\Omega$ is cyclic in $Q \mathscr{H}$ for the reduced algebra $\mathfrak{R}(\mathcal{O})_{Q}^{\prime}$ and for the reduction by $Q$ of the von Neumann algebra generated by $\tilde{\mathfrak{R}}$ and $Q$ - in symbols: $(\mathfrak{R}(\tilde{\mathfrak{R}}, Q))_{Q}$ - which obviously is a subalgebra of $\mathfrak{R}(\mathcal{O})_{Q}^{\prime}$ (for the second statement note that $\Omega$ is cyclic for the set $\{Q \tilde{R} Q\}$ by Lemma 2.4).

But as now $\Omega$ is cyclic for the finite algebra $\mathfrak{R}(\mathcal{O})_{Q}$ in $Q \mathscr{H}$, both algebras $\mathfrak{R}(\mathcal{O})_{Q}^{\prime}$ and $(\Re(\tilde{R}, Q))_{Q}$ must be finite; as they have both $\Omega$ as cyclic and separating vector in $Q \mathscr{H}$ we have: $\mathfrak{R}(\mathcal{O})_{a}^{\prime}=(\mathfrak{R}(\tilde{\mathfrak{R}}, Q))_{Q}$ by Kadison's result $([6]$, Lemma III.2).

2) Next choose $\mathcal{O}_{1}$ open, $\mathcal{O}_{1} \subset \mathcal{O}$, in such a way that $\mathcal{O}_{1, \boldsymbol{a}} \subset \mathcal{O}$ for $\boldsymbol{a}$ from some neighborhood of $\{0\}$ in $\mathbb{R}^{n}$; if $Q_{1}$ denotes the projection onto $\overline{\mathfrak{R}\left(\mathcal{O}_{1}\right) \Omega}$ we have by isotony: $Q_{1, \boldsymbol{a}} \leqq Q$ for all $\boldsymbol{a}$ from the given neighborhood; a standard application of spectrum condition via the "edge of the wedge theorem" yields: $Q_{1, a} \leqq Q$ for all $a \in \mathbb{R}^{n}$.

This means that for arbitrary $A \in \mathfrak{R}\left(\mathcal{O}_{1}\right)$ and arbitrary $\boldsymbol{a} \in \mathbb{R}^{n}$ we have:

$$
Q U(\boldsymbol{a}) A \Omega=U(\boldsymbol{a}) A \Omega .
$$

Now choose $\boldsymbol{r} \in \mathbb{R}^{n}$ such that $\mathfrak{R}_{\boldsymbol{r}}=\mathfrak{R}\left(\mathcal{O}_{1, r}\right)$ is contained in $\tilde{\mathfrak{R}}^{\prime} \cap \mathfrak{R}(\mathcal{O})^{\prime}$; take $A \in \mathfrak{R}_{\boldsymbol{r}}$ then $Q A Q \in \mathfrak{R}(\mathcal{O})_{Q}^{\prime}$; choose $C_{1}, C_{2} \in \mathfrak{R}(\mathcal{O})$ and $B=Q \tilde{B} Q$, where $\tilde{B} \in \tilde{\mathfrak{R}}$, then we get:

$$
\begin{aligned}
& \left(C_{1}, \Omega,[B, Q A Q] C_{2} \Omega\right) \\
& =\left(C_{1} \Omega, B Q A Q C_{2} \Omega\right)-\left(C_{1} \Omega, Q A Q B C_{2} \Omega\right) \\
& =\left(C_{1} \Omega, \tilde{B} C_{2} Q A \Omega\right)-\left(C_{1} Q A^{*} \Omega, \tilde{B} C_{2} \Omega\right) .
\end{aligned}
$$


By (3.1) we may eliminate $Q$ in the last two terms, thus:

$$
\left(C_{1} \Omega,[B, Q A Q] C_{2} \Omega\right)=\left(C_{1} \Omega,[\tilde{B}, A] C_{2} \Omega\right)=0 .
$$

Therefore $Q A Q$ commutes with all $Q \tilde{B} Q$ in $Q \mathscr{H}$ and consequently with arbitrary products of such operators; but these products just generate $(\Re(\tilde{R}, Q))_{Q}$ and so according to 1 ) we must have $Q A Q$ in the center of $\mathfrak{R}(\mathcal{O})_{Q}^{\prime}$ for all $A \in \mathfrak{R}$. From this we get - choosing arbitrary $A, B \in \mathfrak{R}_{r}$-:

$$
0=(\Omega,[Q A Q, Q B Q] \Omega)=(\Omega, A B \Omega)-(\Omega, B A \Omega)
$$

and so, if $\mathfrak{R}(\mathcal{O})$ were finite we had $\Omega$ as a trace vector for $\mathfrak{R}_{r}$.

3) Now let $Q_{1}$ as above, then $\mathfrak{R}\left(\mathcal{O}_{1}\right)_{Q_{1}}$ is finite with $\Omega$ as trace vector by 2 ) and thus by [15], p. 90], we have $\mathfrak{R}\left(\mathcal{O}_{1}\right)_{Q_{1}}^{\prime}$ finite with $\Omega$ as trace vector, too.

Choose now $\mathcal{O}_{2} \mathrm{CO}_{1}$ such that $\mathcal{O}_{2, \boldsymbol{a}} \subset \mathrm{O}_{1}$ for $\boldsymbol{a}$ from a (possibly different from the first one) neighborhood of $\{0\}$ in $\mathbb{R}^{n}$; then for appropriate $s \in \mathbb{R}^{n}$ we have:

$$
\mathfrak{R}\left(\mathcal{O}_{2, s}\right) \subset \tilde{\mathfrak{R}} \subset \mathfrak{R}\left(\mathcal{O}_{1}\right)^{\prime} \text {; } \quad \text { i.e. for } A \in \tilde{\mathfrak{R}} \text { and } B \in \mathfrak{R}\left(\mathcal{O}_{2, s}\right)
$$

we have:

$$
\left(\Omega, Q_{1} A Q_{1} Q_{1} B Q_{1} \Omega\right)=\left(\Omega, A Q_{1} B \Omega\right)=(\Omega, A B \Omega)
$$

as $Q_{1} B \Omega=B \Omega$ by the same argument as in 2). But from this it follows that:

$$
(\Omega, A B \Omega)=(\Omega, B A \Omega)
$$

because $\Omega$ was a trace vector for $\mathfrak{R}\left(\mathcal{O}_{1}\right)_{Q_{1}}^{\prime}$; but (3.6) in turn means that $B \in \tilde{\mathfrak{R}}$ is in $\tilde{\mathfrak{R}}^{\Sigma}$ - according to Theorem 2.2 this implies that $\mathfrak{R}\left(\mathcal{O}_{2}\right)$ is one dimensional, a contradiction. q.e.d.

Remark 3.2. The result raises the general question as to whether nontriviality of all $\mathfrak{R}(\mathcal{O})$ can replace weak additivity on a wider scale; at present we don't have a satisfactory answer to it and so this question remains open.

Let us next consider two different sets $\mathcal{O}_{i}, i=1,2$, where we assume from now on $\mathcal{O}_{1} \times \mathcal{O}_{2}$. In order to to express the supposed independence of events in $\mathcal{O}_{1}$, resp. $\hat{O}_{2}$, people have defined a number of concepts going beyond pure locality. We shall consider two of these concepts and therefore we define:

Definition 3.3. A) Two von Neumann algebras $\mathfrak{R}\left(\mathcal{O}_{1}\right), \mathfrak{R}\left(\mathcal{O}_{2}\right)$ with

$\mathcal{O}_{1} \times \mathcal{O}_{2}$ have the Schlieder property iff $A_{1} \cdot A_{2}=0$

implies $A_{1}=0$ or $A_{2}=0$.

B) The two algebras will be called strictly local iff for an arbitrary projection $P \in \mathfrak{R}_{1}$ and any normal state $\varrho_{\varphi}$ on $\mathfrak{R}_{2}$ we can find $\Psi \in P \mathscr{H}$ such that $\varrho_{\psi} / \mathfrak{R}_{2}=$ $\varrho_{\varphi} / \mathfrak{R}_{2}\left(\Omega\right.$ is separating for all $\mathfrak{R}_{i}$, so all normal states are vector states).

The Schlieder property (SP) is important being equivalent to statistical independence of $C^{*}$-algebras $([16])$; strict locality (SL) tells us that no measurement in $\mathcal{O}_{1}$ can give any information concerning any observable in $\mathcal{O}_{2}$. For a more detailed discussion of these concepts see [17-19] and references quoted there.

For both of the previously defined properties there is - as far as we know only one proof in each case deriving the property from general principles; Schlieder's proof when adapted to the case of open $\mathcal{O}$, however, needs the extra assump- 
tion $\mathcal{O}_{1, t} \times \mathcal{O}_{2}$ for $|t| \leqq \delta, \delta>0$ arbitrary, thus leaving open the question for arbitrary spacelike $\mathcal{O}_{1}, \mathcal{O}_{2}$; Kraus' proof of SL ([18]) needs too many assumptions which cannot be considered as physically equally well founded as the standard ones and which one would therefore like to eliminate altogether at least in particular cases.

In order to make a step forward in this matter we first define:

Definition 3.4. Let $\mathcal{O}_{1}, \mathcal{O}_{2}$ be two b.o.r. in $\mathbb{R}^{n}$ with $\mathcal{O}_{1} \times \mathcal{O}_{2}$. The algebras $\mathfrak{R}\left(\mathcal{O}_{i}\right)$, $i=1,2$, are lightlike disjoint iff $\mathfrak{R}_{1}$ may be imbedded into some lightlike monotone algebra $\mathfrak{R} \subset \mathfrak{R}_{2}^{\prime}$.

Then we can state:

Theorem 3.5. If $\mathfrak{R}_{i}, i=1,2$ are lightlike disjoint they obey SP and SL under the assumptions of Section 1.

Proof. a) For SP the assertion is an immediate consequence of Definition 3.4 and the classical result of von Neumann for factors and their commutants ([20], p. 30).

b) By assumption we can find $\mathfrak{R} \in \mathscr{A}$ with $\mathfrak{R}_{1} \subset \mathfrak{R} \subset \mathfrak{R}_{2}^{\prime}$; then by known theorems (here we need $\mathscr{H}$ to be separable) every projection $P \in \mathfrak{R}_{1}$ is infinite and therefore equivalent to $I$ in $\mathfrak{R}$ (a type III factor!); therefore there exists a partially isometric operator $U \in \Re$ such that: $U^{*} U=I, U U^{*}=P$.

As $\Omega$ is separating for all algebras involved (here we assume of course $\varepsilon$ ) again) every normal state $\sigma$ on $\mathfrak{R}^{\prime}$ is a vector state $\sigma_{\Phi}, \Phi \in \mathscr{H}$. But by construction $\Phi$ and $U \Phi$ yield the same state on $\mathfrak{R}^{\prime}$, therefore we have also $\sigma_{\Phi} / \mathfrak{R}_{1}=\sigma_{U \Phi} / \mathfrak{R}_{2}$. q.e.d.

Now this result again raises the question as to whether its limitation to lightlike disjointness is merely accidental or whether there is something real behind it. In order to support the conjecture that the latter may be true we state:

Lemma 3.6. Let $\underline{\mathcal{O}}_{1}, \underline{\mathcal{O}}_{2}$ be disjoint open sets in $\mathbb{R}^{n-1}$ (to be taken as the hyperplane $\{t=0\})$ and let $\mathcal{O}_{1}, \mathcal{O}_{2}$ denote their respective causal shadows. Then lightlike disjointness of $\mathfrak{R}\left(\mathrm{O}_{1}\right)$ and $\mathfrak{R}\left(\mathrm{O}_{2}\right)$ is equivalent to existence of a $(n-2)$-dimensional hyperplane in $\mathbb{R}^{n-1}$ which separates $\mathcal{O}_{1}$ and $\mathcal{O}_{2}$.

The proof is very easy and will not be given here. In such a situation consider now the two backward lightcones from $\mathcal{O}_{1}$ resp. $\mathcal{O}_{2}$. Our lemma then tells us that each of the $\mathcal{O}_{i}$ receives influences from an arbitrarily remote past which cannot disturb events in the other region. This seems to correspond very well to the intuitive idea of having no correlation whatsoever between events in $\mathcal{O}_{1} \operatorname{resp} . \mathcal{O}_{2}$.

Our results allow immediate generalization to the case of a finite number of commuting algebras $\left\{\Re\left(\mathcal{O}_{i}\right)\right\}_{1}^{n}$ if these algebras lie spacelike to each other in such a way that our argument can be applied to each pair $\left(\bigcup_{i=1}^{k} \mathfrak{R}\left(\mathcal{O}_{i}\right)\right)^{\prime \prime}$ and $\mathfrak{R}\left(\mathcal{O}_{k+1}\right)$ successively. This is, however, not sufficient to cover all relevant cases but gives support to the conjecture that SP and SL may be true under even more general circumstances.

Acknowledgements. The author is grateful for helpful discussions with Dr. Brüing and Dr. EnB as well as for the numerous helpful suggestions of Prof. Haag and Dr. Buchholz concerning the final version of this paper. 


\section{References}

1. Emch, G.: Algebraic methods in statistical mechanics and QFT. New York: Wiley Interscience 1972

2. Buchholz, D.: Collision theory for massless fermions. Preprint, CERN TH 1955

3. Driessler, W.: Thesis (to appear)

4. Araki, H.: Progr. Theor. Phys. 32, 956-965 (1964)

5. Dell'Antonio, G. F.: Commun. math. Phys. 9, 81-117 (1968)

6. Kadison, R.V.: Journ. Math. Phys. 4, 1511-1516 (1963)

7. Guenin, M., Misra, B.: Nuovo Cim. 30, Ser. 10, 1272-1290 (1963)

8. Pedersen, G., Takesaki, M.: Acta. Math. 130, 53-87 (1973)

9. Takesaki, M.: Lecture Notes 128. Berlin Heidelberg New York: Springer 1970

10. Connes, A.: Ann. Scient. de l'Ecole Norm. Sup. 4th series, Vol. 6, 133-252 (1973)

11. Doplicher, S., Haag, R., Roberts, J. E.: Commun. math. Phys. 13, 1-23 (1969)

12. Maison, D.: Commun. math. Phys. 10, 48-51 (1968)

13. Sadowski, P., Woronowicz, S. L.: Rep. Math. Phys. 2, 113-120 (1971)

14. Misra, B.: Helv. Phys. Acta 38, 189-206 (1965)

15. Dixmier, J.: Les algèbres d'operateurs dans l'espace Hilbertien. Paris: Gauthier Villars 1969

16. Roos, H.: Commun. math. Phys. 16, 238--246 (1970)

17. Schlieder, E.: Commun. math. Phys. 13, 215-225 (1969)

18. Kraus, K.: Zeitschr. f. Phys. 181, 1-12 (1964)

19. De Facio, B.: Causal independence in algebraic QFT (preprint)

20. von Neumann, J.: Collected works, Vol. III. New York: Pergamon Press 1961

Communicated by R. Haag 
